\title{
Component Isolation for Multi-component Signal Analysis Using a Non-parametric Gaussian Latent Feature Model
}

\author{
Yang Yang, ${ }^{\mathrm{a}, \mathrm{b}}$, Zhike Peng ${ }^{\mathrm{a}}$, Xingjian Dong ${ }^{\mathrm{a}}$, Wenming Zhang ${ }^{\mathrm{a}}$, David A. \\ Clifton ${ }^{\mathrm{b}}$ \\ ${ }^{a}$ Department of Mechanical Engineering, Shanghai Jiao Tong University, Shanghai, \\ China \\ ${ }^{b}$ Institute of Biomedical Engineering, Department of Engineering Science, University of \\ Oxford, Oxford, UK
}

\begin{abstract}
Abstract- A challenge in analysing non-stationary multi-component signals is to isolate nonlinearly time-varying signals especially when they are overlapped in time and frequency plane. In this paper, a framework integrating time-frequency analysis-based demodulation and a non-parametric Gaussian latent feature model is proposed to isolate and recover components of such signals. The former aims to remove high-order frequency modulation (FM) such that the latter is able to infer demodulated components while simultaneously discovering the number of the target components. The proposed method is effective in isolating multiple components that have the same FM behavior. In addition, the results show that the proposed method is superior to filter-based isolation, with user-defined bandwidth, in recovering the amplitude and phase of superimposed components.
\end{abstract}

Keywords: Time-frequency analysis, non-parametric latent feature model, time series, continuous phase modulation, component extraction.

\section{Introduction}

In many applications encountered in practice, non-stationary multi-component signals consist of various non-linearly frequency-modulated (FM) components. The latter convey important information concerning the non-stationary process that assumedly generates the signals. A critical signal processing 
task is to isolate such components, where this is complicated by the complex time-frequency structures of the signals; e.g., the components intersect in the time-frequency domain. Empirical mode decomposition (EMD) is known for decomposing a signal into a series of intrinsic mode functions (IMF) [6], each of which is assumed to be a mono-component. However, EMD is incapable of dealing with intersected components or broad-bandwidth nonlinear FM components. Filter- or mask-based methods in the time-frequency domain could cope with such components [1], though a substantial challenge is to determine bandwidth or masking size to capture local features. A trade-off of using filters or masks lies in recovering the intersected components; i.e., one is fully recovered while the other will be broken down and cannot be recovered at the point at which components cross. Widely-studied component separation methods such as thee Hough transform [2], singular value decomposition [3], and energy separation [4] perform well in the case of intersected stationary components, though the number of the target components needs to be defined in advance.

The non-parametric Gaussian latent feature model (NGLF) [12, 13, 14, $15,16,17]$ is known for its capacity to infer latent features without prior knowledge. The components of a multi-component signal can be considered as being latent features of the signal; the NGLF thereby holds significant potential for improved component isolation. The NGLF is superior to independent component analysis [7, 8, 9] and non-negative matrix factorisation [10], which are two well-studied models for automatic component isolation, in the case that the number of the latent features is unknown. However, the latter methods assume that the latent feature is static in the observations, while time-frequency patterns of FM components vary with time. A challenge exists in how to apply the NGLF to the identification of FM components. A straightforward solution is to project the FM components into a space where the components are stationary so that the NGLF is able to resolve the latent features.

In this paper, we propose an integrated framework that combines i) a time-frequency analysis-based demodulation and ii) the NGLF. The demodulation technique assumes a frequency-modulated signal model with the phase modulating by a high-order function of time $[18,19]$. To acquire the parameters of an arbitrary phase function, we adapt the parametric time-frequency transform (PTFT) [5]. The latter was proposed as a time-frequency domain analysis method that is capable of using an arbitrary phase transform kernel to capture the local time-frequency pattern of a non-stationary signal, and to 
obtain a time-frequency representation with enhanced energy concentration. An iterative parameter estimation scheme associated with the PTFT provides the basis for demodulating various multi-component signals. In [25], we applied the PTFT and a simple band-pass filter for component extraction, though the filter resulted in attenuation at the edges of components and amplitude distortion at the cross-point between intersecting components. In [23], we integrated PTFT with singular value decomposition (SVD), but we note that the SVD strongly relies on the accuracy of the estimation of the demodulation. In addition, it can neither deal with multiple components with the same frequency modulation nor with local components. In [24], a joint-refinement post-processing step was used to recover the amplitude of intersected components, though it assumed that the component is known and the estimation for all components cannot reach the best simultaneously. The framework described in this paper provides alternative solutions to cope with the above limitations in component isolation. In the proposed framework, the PTFT aims to remove the high-order FM in components so that the NGLF is able to infer the demodulated components automatically without defining the number of the components. WE show that the proposed method is effective in isolating multiple components with the same FM behavior at one time. In addition, the results show that the proposed method is superior to filter-based isolation in recovering the amplitudes of superimposed components.

The remainder of this paper is organised as follows: section II introduces a signal model and the underlying principles of the NGLF and PTFT; section III provides the details of the proposed framework and a demonstration on an exemplar signal. Section IV provides analysis results of applying the proposed method with both simulated and experiment signals. Finally, section V draw conclusions.

\section{Theoretical background}

\subsection{Signal model}

We assume a multi-component signal $S(t) \in \mathbb{R}^{1}$ consisting of $L$ components $s(t)$,

$$
S(t)=\sum_{l}^{L} s_{l}(t)
$$


with

$$
s(t)=A(t) \exp \{-i \phi(t)\}
$$

where $\sqrt{i}=-1$. $A(t)$ and $\phi(t)$ are time-varying amplitude and phase, respectively. Eq.2 is an analytic form of signal components. Instantaneous frequency (IF) is a measurement of time-frequency pattern defined for nonstationary signal as,

$$
I F(t)=\frac{1}{2 \pi} \frac{\mathrm{d}}{\mathrm{d} t} \phi(t)
$$

which assumes that the phase function $\phi(t)$ is differentiable. Noted that this definition is for mono-component signals, it is also valid for individual component of multi-component signals.

A signal with finite time support can be cast into a set of segments with overlap. For example, the multi-component signal $S(t)$ is divided into a set of overlapped segments with the length of $D$; i.e., $\mathbf{X}=\left\{x^{(1)}, \ldots x^{(N)}\right\}, x^{i} \in \mathbb{R}^{D}$.

We assume component set $\mathbf{A}=\left\{a^{(1)}, \ldots, a^{(K)}\right\}, a^{j} \in \mathbb{R}^{D}$ is responsible for generating the data $\mathbf{X}$,

$$
\mathbf{X}=\mathbf{W}^{T} \mathbf{A}
$$

where $\mathbf{W}$ is a binary matrix with $w_{i j}=[0,1]$ denoting if the $i$ th example contains the $j$ th feature. $K$ stands for the number of features and $K \geq L$. The equality holds if and only if the components are stationary.

\subsection{Parametric time-frequency transform}

The parametric time-frequency transform (PTFT) is a general formula that uses a kernel function to transform a time-domain signal into the timefrequency domain [21]. An advantage of the PTFT is that it applies adaptive kernels that approximate time-frequency patterns of analysed signals to improve the readability of their time-frequency representations. It is especially effective for non-stationary signals with various shapes of IF trajectory in the time-frequency domain.

The PTFT is defined as

$$
\operatorname{PTFT}(t, \omega ; \mathbf{P})=\int_{-\infty}^{\infty} \bar{s}(\tau) h(\tau-t) \exp \{-i \omega \tau\} d \tau
$$

with

$$
\begin{array}{r}
\bar{s}(\tau)=s(\tau) \Phi^{R}(\tau ; \mathbf{P}) \Phi^{S}\left(\tau ; \mathbf{P}, t_{0}\right) \\
\Phi^{R}(\tau ; \mathbf{P})=\exp \left\{-i \int K(\tau ; \mathbf{P}) d \tau\right\} \\
\Phi^{S}\left(\tau ; \mathbf{P}, t_{0}\right)=\exp \left\{i \tau K\left(t_{0} ; \mathbf{P}\right)\right\}
\end{array}
$$


where $\Phi^{R}(\tau ; \mathbf{P})$ and $\Phi^{S}\left(\tau ; \mathbf{P}, t_{0}\right)$ are frequency rotation and shifting operators, respectively; $\omega$ is frequency; $h(t)$ is a window function; $K(\tau ; \mathbf{P})$ denotes the kernel function that can be integrated with the time given the parameter P. $t_{0}$ is the central time within an interval of evaluation $\tau$. The goal of the PTFT is to apply the frequency rotation operator, $\Phi^{R}(\tau ; \mathbf{P})$, to remove the time variation of frequency across all frames of the signal within interval $\tau$, and then use the frequency shift rotation operator, $\Phi^{S}\left(\tau ; \mathbf{P}, t_{0}\right)$, to carry the time-invariant frequency spectrum back to the centre of the each frame.

As counterparts of the PTFT, non-parametric time-frequency transforms with a signal-independent window function, (e.g., the short-time Fourier transform and the continuous wavelet transform), result in smearing of energy in the time-frequency representation in the case of a non-stationary signal. As a result, the readability of the time-frequency representation will be reduced due to the unsatisfied energy concentration. On the other hand, the PTFT offers twofold benefits: 1) concentration the otherwise-smeared energy in the grid of time-frequency representation; 2) optimising parameters in terms of energy concentration in the rotated frequency spectrum.

\subsection{Non-parametric Gaussian latent feature model}

The non-parametric Gaussian latent feature (NGLF) model is a statistical model that assumes that the examples are generated by a Gaussian distribution with covariance and mean given by a linear combination of latent features and binary weights. The latent features and binary weights are assumed to have priors that are a Gaussian process (GP) and Indian buffet process (IBP), respectively. The purpose of the model is to infer the posterior of the binary weights given the data, meanwhile the latent features are obtained given the data and the binary weights. In our study, the latent features correspond to the components and noise, while the binary weights denote the presence or absence of those components.

The model assumes that a $d$-dimensional vector of an example $x^{(i)}$ is generated from a Gaussian distribution with mean $\mathbf{w}_{\mathbf{i}} \mathbf{A}$ and covariance matrix $\sum_{X}=\sigma_{X}^{2} \mathbf{I}$, where $\mathbf{w}_{\mathbf{i}}$ is a $K$-dimensional binary vector, and $\mathbf{A}$ is a $K \times D$ matrix (as shown in Fig.1).

According to Bayes' theorem, the posterior distribution over $\mathbf{W}$ can be obtained via

$$
p\left(\mathbf{W} \mid \mathbf{X}, \sigma_{X}, \sigma_{A}\right) \propto p\left(\mathbf{X} \mid \mathbf{W}, \sigma_{X}, \sigma_{A}\right) p(\mathbf{W})
$$




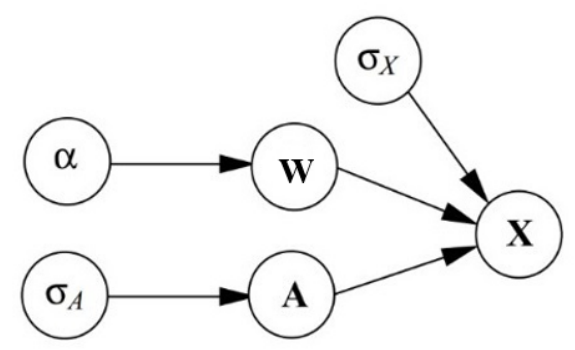

Figure 1: Graphical model for the NFL [20]

where $\sigma_{X}$ is the covariance of observation matrix $\mathbf{X}$. The first term in the right-hand side of Eq.(7), the likelihood of $p\left(\mathbf{X} \mid \mathbf{W}, \sigma_{X}, \sigma_{A}\right)$, is computed by marginalising the unknown feature $\mathbf{A}$,

$$
p\left(\mathbf{X} \mid \mathbf{W}, \sigma_{X}, \sigma_{A}\right)=\int p\left(\mathbf{X} \mid \mathbf{W}, \mathbf{A}, \sigma_{X}\right) p\left(\mathbf{A} \mid \sigma_{A}\right) \mathrm{d} \mathbf{A}
$$

where the probability of $\mathbf{X}$ given $\mathbf{W}, \mathbf{A}$, and $\sigma_{X}$ is modelled as a multivariate Gaussian distribution as aforementioned. The probability of $\mathbf{A}$ given $\sigma_{A}$ is also a multivariate Gaussian distribution, $M N\left(0, \sigma_{A}^{2} \mathbf{I}\right)$. Therefore, Eq.(8) is computed via

$$
\begin{array}{r}
p\left(\mathbf{X} \mid \mathbf{W}, \sigma_{X}, \sigma_{A}\right)=\frac{1}{(2 \pi)^{N D / 2} \sigma_{X}^{N-K} \sigma_{A}^{K D}|\mathbf{H}|^{D / 2}} \\
\quad \exp \left\{-\frac{1}{2 \sigma_{X}^{2}} \operatorname{tr}\left(\mathbf{X}^{T}\left(\mathbf{I}-\mathbf{W H}^{-1} \mathbf{W}^{T}\right) \mathbf{X}\right)\right\}
\end{array}
$$

with

$$
\mathbf{H}=\mathbf{W}^{T} \mathbf{W}+\frac{\sigma_{X}^{2}}{\sigma_{A}^{2}} \mathbf{I}
$$

where $\operatorname{tr}()$ is the trace of a matrix.

The prior probability of $\mathbf{W}$, the second term in the right-hand side of Eq.(7), is assumed to follow the IBP, which allows the matrix to have an unbounded number of columns. A generative process for a binary weight matrix is defined as,

$$
\pi_{j}\left|\alpha \sim \operatorname{Beta}\left(\frac{\alpha}{K}, 1\right) \quad w_{i j}\right| \pi_{j} \sim \operatorname{Ber}\left(\pi_{j}\right)
$$

Each $w_{i j}$ follows a Bernoulli distribution and is independent of all other assignments, conditioned on $\pi_{j}$. The $\pi_{j}$ follows a Beta distribution and is 
generated as being independently conditioned on $\alpha$. By interpreting the generative process using an IBP, the probability of each entry in the binary matrix $p\left(w_{i j} \mid \mathbf{w}_{-i, j}\right)$ can be evaluated as,

$$
p\left(w_{i j}=1 \mid \mathbf{w}_{-i, j}\right)=\frac{m_{-i, j}+\frac{\alpha}{K}}{N+\frac{\alpha}{K}}
$$

where $m_{j}=\sum_{i=1}^{N} w_{i j}$. The marginal probability of the binary matrix $\mathbf{W}$ is

$$
p(\mathbf{W} \mid \alpha)=\prod_{k=1}^{K} \frac{\frac{\alpha}{K} \Gamma\left(m_{k}+\frac{\alpha}{K}\right) \Gamma\left(N-m_{k}+1\right)}{\Gamma\left(N+1+\frac{\alpha}{K}\right)}
$$

where $\Gamma()$ is the gamma function.

In aggregate, the full conditional distribution for $w_{i k}$ is given as

$$
p\left(w_{i j} \mid \mathbf{X}, \mathbf{W}_{-i, j}, \sigma_{X}, \sigma_{A}\right) \propto p\left(\mathbf{X} \mid \mathbf{W}, \sigma_{X}, \sigma_{A}\right) p\left(w_{i j} \mid \mathbf{w}_{-i, j}\right)
$$

As the IBP assumes that the rows and columns are exchangeable, the generative process results in a group of binary matrices; these matrices share a unique left-order-form (lof) matrix which rearrange the rows and columns to the top and left according to the number of non-zero entries. The probability of a particular lof-equivalence class of binary matrices, $[\mathbf{W}]$, is

$$
p(\mathbf{W})=\frac{K !}{\prod_{h=0}^{2^{N}-1} K_{h} !} \prod_{k=1}^{K} \frac{\frac{\alpha}{K} \Gamma\left(m_{j}+\frac{\alpha}{K}\right) \Gamma\left(N-m_{j}+1\right)}{\Gamma\left(N+1+\frac{\alpha}{K}\right)}
$$

where $K_{h}$ is the count of the number of features with full history $h$. The history of feature $j$ for object $i$ is defined to be $\left(w_{1 j}, \ldots, w_{(i-1) j}\right)$, while the full history of feature $j$, without a specified object, is referred to as $\left(w_{1 j}, \ldots, w_{N j}\right)$. Taking the limit for this probability of $\mathbf{W}$ as $K \rightarrow \infty$ as

$$
\begin{gathered}
\lim _{K \rightarrow \inf } p(\mathbf{W})= \\
\frac{\alpha^{K_{+}}}{\prod_{h=1}^{2^{N}-1} K_{n} !} \exp \left(-\alpha H_{N}\right) \prod_{k=1}^{K_{+}} \frac{\left(N-m_{j}\right) !\left(m_{j}-1\right) !}{N !}
\end{gathered}
$$

where $K_{+}$is the number of columns for which $m_{k}>0$ and $H_{N}=\sum_{j=1}^{N} \frac{1}{j}$ is the $N$ th harmonic number. Considering an infinite number of features, the 
probability of $\mathbf{X}$ given $\mathbf{W}, \sigma_{X}$, and $\sigma_{A}$ changes $\mathbf{W}$ and $\mathrm{K}$ in Eq.(9) to be $\mathbf{W}_{+}$ and $K_{+}$, where $\mathbf{W}_{+}$is the corresponding submatrix of the active features.

To perform inference with $\mathbf{W}$, a heuristic Gibbs sampling algorithm is used to draw examples via Eq.(14), using Eq.(12) and (9) to compute $p\left(w_{i j} \mid \mathbf{w}_{-i, j}\right)$ and $p\left(\mathbf{X} \mid \mathbf{W}, \sigma_{X}, \sigma_{A}\right)$, respectively. Here, $\sigma_{A}$ and $\sigma_{X}$ are sampled via a Metropolis-Hastings step; the acceptance of proposed values is determined by evaluating $p\left(\mathbf{X} \mid \mathbf{W}, \sigma_{X}, \sigma_{A}\right)$ from Eq.(9). For each new component, $p\left(\mathbf{X} \mid \mathbf{W}, \sigma_{X}, \sigma_{A}\right)$ is also computed, and where the prior on the number of new components is $\operatorname{Poisson}\left(\frac{\alpha}{N}\right)$. The latent features are calculated as,

$$
E[\mathbf{A} \mid \mathbf{W}, \mathbf{X}]=\left(\mathbf{W}^{T} \mathbf{W}+\frac{\sigma_{X}^{2}}{\sigma_{A}^{2}} \mathbf{I}\right)^{-1} \mathbf{W}^{T} \mathbf{X}
$$

\section{Component isolation framework}

\subsection{Stationary component isolation}

The NGLF aims to reveal latent features that are globally stationary . The underlying principle of the NGLF for component isolation is illustrated in

Fig.2. For a two-component signal with one stationary component, the time series is first cast into a series of sequentially overlapped segments. Next, an NGLF model takes the segments as input and obtains latent features. The obtained first latent feature corresponds to the stationary component. The other components may contain different segments of the non-stationary component and noise. This is because the NGLF considers non-stationary components to be local features. Then, the stationary component can be reconstructed using the first latent feature, meanwhile the other component can be obtained by removing the former from the original signal.

If there are multiple stationary components exist, the NGLF model will consider them as being one latent feature due to the co-occurrence. In this case, these co-occurred stationary components will be extracted from the analysed signal simultaneously. Such characteristics can also be used to distinguish stationary components from others; i.e., close-spaced quasistationary components and modulated noise.

\subsection{Non-stationary component isolation}

To isolate non-stationary components from a signal without stationary components, the PTFT and an associated parameter estimation scheme are 
Two-component signal

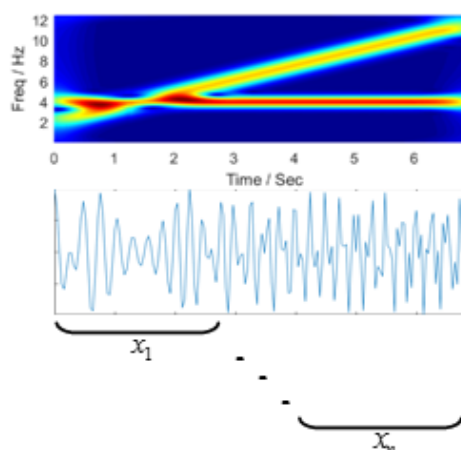

$\Rightarrow\left[\begin{array}{c}x_{1} \\ \vdots \\ x_{n}\end{array}\right]$

First latent component

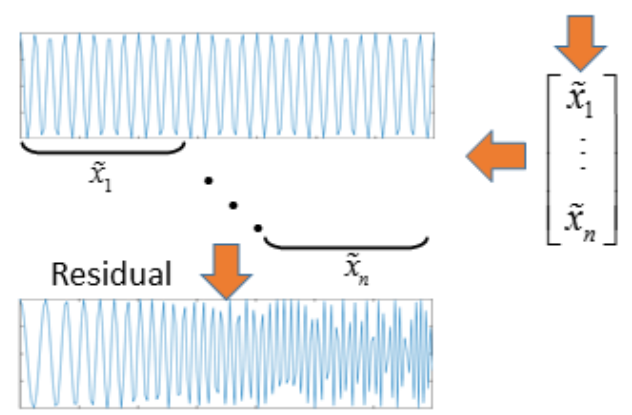

Figure 2: Flowchart of NLFM applied to a univariate signal $s(t)$ 
used to pre-process the considered signal. The kernel function of the PTFT can be (for example) a polynomial, a Fourier series, or spline functions. In the investigation described in this paper, we use a polynomial kernel as,

$$
K\left(t ; p_{0}, \ldots p_{m}\right)=p_{0}+p_{1} t+p_{2} t^{2} \ldots+p_{m} t^{m}
$$

where $m$ is the order of the polynomial function. With the obtained timefrequency representation $\operatorname{PTFT}(t, \omega ; \mathbf{P})$, the IF of the signal $s(t)$ is estimated by evaluating the peak along the time axis,

$$
\tilde{I F}(t)=\underset{t}{\operatorname{argmax}}|\operatorname{PTFT}(t, \omega ; \mathbf{P})|^{2}
$$

The parameter estimation scheme iteratively approximates IF via Eq.(19) and (18). This will only be accurate when the IF is well approximated. The time-frequency representation will be gradually improved when the estimated parameters become more accurate. Ideally, the estimated parameters enable the targeted component to be demodulated completely, though the parameter estimation process corrupted by noise in the original signal and interfered components that are intersected or which have high energy, typically results in incomplete demodulation. In this case, more than one of the latent features obtained by the NGLF model might include the considered component. Hence, the unwanted components in the latent feature set require us to delete them. The latent feature selection is performed by measuring the ratio of peak power over bandwidth in their frequency spectrum, which is defined as

$$
r=\frac{\underset{\omega}{\operatorname{argmax}} F\left[a^{(l)}\right]}{\underset{\omega}{\operatorname{argmax}}\left(F\left[a^{(l)}\right]>\delta\right)-\underset{\omega}{\operatorname{argmin}}\left(F\left[a^{(l)}\right]>\delta\right)}
$$

where $F$ denotes the Fourier transform operator, $\delta$ is a user-defined value, which we use as being $30 \%$ of the magnitude of peak $\max F\left[a^{(l)}\right]$. Here, $a^{(l)}$ is the $l$ th latent feature. As long as the value of $r$ falls below a pre-defined threshold, the latent feature will be discarded while the preserved latent features will be used to reconstruct the intersected component.

The details of the proposed framework are provided in Algorithm 1. For stationary component isolation, the first loop for estimating phase parameter should be omitted. We note that the NGLF model is also able to isolate an asymptotic component whose amplitude is assumed to change more slowly than frequency. 
Data: $S(t)$, window length $d$, maximum sampling times $\mathbf{T}_{\max }$, terminating threshold $\delta$, threshold of ratio $\epsilon$

Result: Reconstructed components and residual

Initialization $i=1, \tilde{I F_{0}}=0, \mathbf{P}_{0}=0, \sigma_{X}, \sigma_{A}$;

while $\left|\tilde{I} F_{i}(t)-\tilde{I F} F_{i-1}\right|<\delta, r S(t)=S(t)$ do

perform the PTFT via Eq.(5) and (6);

estimate IF of $s_{l}(t)$ via Eq.(19);

fit Eq. (18) for the estimated IF;

$i=i+1$;

obtain kernel parameter $\mathbf{P}_{i}$;

end

Demodulate $r S(t)$ using $\mathbf{P}$ to obtain $S_{d}(t)$;

Cast $S_{d}(t)$ into $\mathbf{X}$;

while $s t<\mathbf{T}_{\max }$ do

Sample $\sigma_{A}^{(s t)}$ using Metropolis-Hastings;

Sample $\sigma_{X}^{(s t)}$ using Metropolis-Hastings;

Sample binary matrix $\mathbf{W}^{(s t)}$ via Eq.(12);

end

Compute A via Eq.(17);

Select latent features with $r>\epsilon$ via Eq.(20), $\mathbf{A} \rightarrow \mathbf{A}^{-}, \mathbf{W} \rightarrow \mathbf{W}^{-}$;

Reconstruct component with $\mathbf{A}^{-}$and $\mathbf{W}^{-}$, and $\mathbf{P}, s_{l}(t) \rightarrow \tilde{s}_{l}(t)$; $r S(t) \leftarrow r S(t)-\tilde{s}_{l}(t)$

Repeat until no more intersected components remain in $r S(t)$;

Algorithm 1: The proposed component isolation framework 
For non-stationary components, the phase parameters are estimated via the PTFT-based iterative parameter estimation method, which are then used for demodulation as described earlier. The demodulated signal is cast as being a data matrix of segments using a sliding window. Each row of the matrix is considered as being an example observation. The NGLF is applied to the data matrix and obtains its weight matrix and latent features via Metropolis-Hastings and heuristic Gibbs sampling. Only those latent features containing the demodulated component are selected by evaluating the ratio in the frequency domain. Accordingly, the dimension of weight matrix is reduced. The selected latent features and binary matrix are then used for reconstructing the demodulated component, which is finally remodulated via the parameters estimated for demodulation. The process will be repeated until no more intersected components remain in the residual of the signal.

\section{Analysis and discussion}

In this section, we present results of analysis using three simulated signals and an experimental signal to illustrate the performance of the proposed method. All the simulated signals are contaminated by additive Gaussian noise with a signal-to-noise rate of $5 \mathrm{~dB}$.

\subsection{Example 1}

A 3-component signal is considered,

$$
S(t)=s_{1}(t)+s_{2}(t)+s_{3}(t)
$$

with

$$
\begin{gathered}
s_{1}(t)=0.5 * \cos \left(2 \pi t^{2}\right) \\
s_{2}(t)=\cos \left[2 \pi\left(12 t-t^{2}\right)\right] \\
s_{3}(t)=0.6 * \cos (2 \pi 6 t)
\end{gathered}
$$

where the IFs of the first two components are linear functions of time; i.e., $2 t$ and $12-2 t$, respectively. The proposed method is compared with linear filtering. The bandwidth of the filter is set to $5-7 \mathrm{~Hz}$. Fig. 3(a) and (b) show the three-component waveforms individually, and their corresponding time-frequency representation. Fig. 3(b) shows the three components are intersected at $t=3 \mathrm{~s}$ with overlapped frequency of $6 \mathrm{~Hz}$. Fig. 3(c) and (d) are reconstructed waveforms of $s_{3}(t)$ using the filter approach and the NGLF 
model, respectively. The former attenuates at both ends and it is enhanced at $t=3 s$, which interferes with the other components at the cross-point. The NGLF model maintains the amplitude feature of $s_{3}(t)$ at these locations, which shows that the proposed approach is able to reconstruct components without loss of amplitude information. Fig. 3(e) and (f) show the timefrequency representations of the residual by removing $s_{3}(t)$ recovered by the filter and the NGLF model, respectively. The region shown by the black square in Fig. 3(e) emphasises that the filter removes the proportion of the residual at the cross-point where the amplitudes of the other components cannot be recovered. For the proposed method, the residual at the cross-point is preserved, leading to accurate reconstruction of the other two components sequentially. In addition, this example shows an advantage of the proposed method in isolating stationary components in an automatic manner, without knowing their frequency.

\subsection{Example 2}

We consider a 3-component signal with components,

$$
\begin{gathered}
s_{1}(t)=\cos \left[2 \pi\left(2 t+0.7 t^{2}\right)\right] \\
s_{2}(t)=\cos (2 \pi 10 t) \text { where } s_{2}=0 \text { if } t>3.5 s \\
s_{3}(t)=\cos (2 \pi 4 t)
\end{gathered}
$$

where $s_{1}(t)$ is a chirping component with a chirping rate of $1.4 \mathrm{~Hz} / \mathrm{s}, s_{2}(t)$ is a local stationary component of $10 \mathrm{~Hz}$ from $0 \mathrm{~s}$ to $3.5 \mathrm{~s}$, and $s_{3}$ is a global stationary component of $4 \mathrm{~Hz}$. Fig.4(a) shows the time-frequency plot of the considered signal, which shows $s_{1}(t)$ is intersected with $s_{3}(t)$ at $t=1.5 \mathrm{~s}$. In this example, the two stationary components are considered concurrently in the NGLF model so they are extracted simultaneously, as shown in Fig. 4(b). Fig. 4(c) shows the residual after removing the reconstructed two stationary components. We note a Gabor-shaped component of $10 \mathrm{~Hz}$ at $t=3.5 \mathrm{~s}$ remains because of phase coupling of $s_{2}$ at $t=3.5 \mathrm{~s}$. In the case

of using the filter to isolate components, two cascade filters are needed to isolate $s_{2}$ and $s_{3}$, respectively, and $s_{1}(t)$ will result in three pieces.

\subsection{Example 3}

Here we consider a 2-component signal consisting of two nonlinear frequencymodulated (FM) components,

$$
S(t)=s_{1}(1)+s_{2}(t)
$$




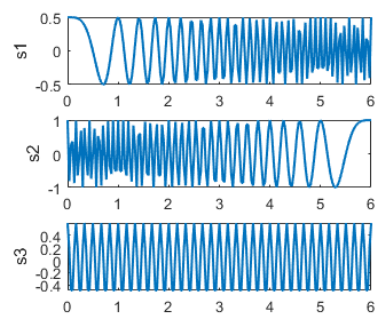

(a)

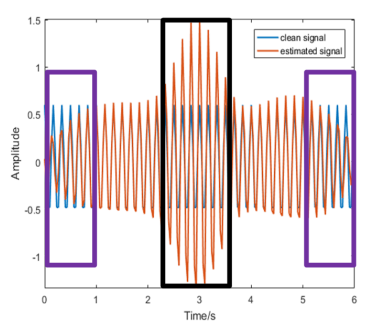

(c)

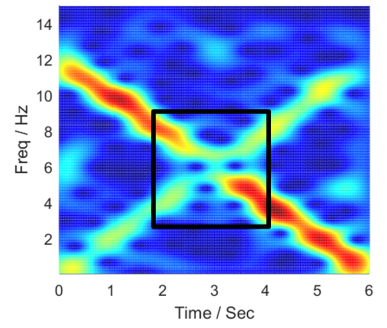

(e)

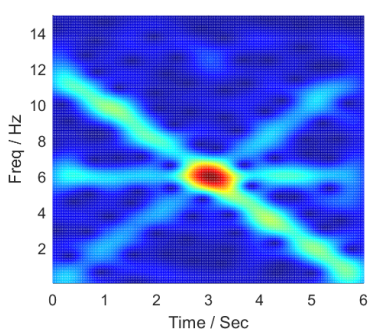

(b)

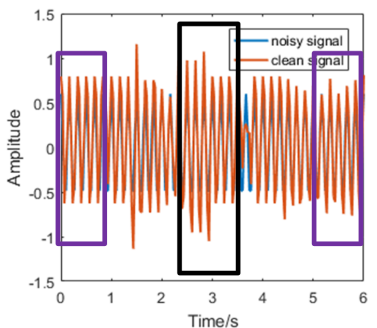

(d)

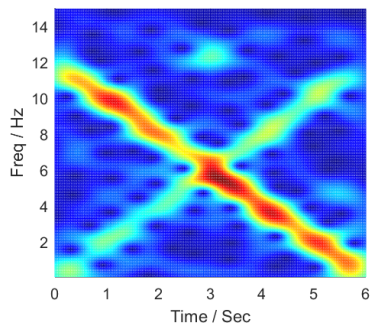

(f)

Figure 3: Demonstration of NLFM for example 1: (a) waveform of $s_{1}(t), s_{2}(t)$ and $s_{3}$; (b) time-frequency plot of $S(t)$; (c) $s_{3}(t)$ obtained by band-pass filter; (d) $s_{3}(t)$ reconstructed by proposed method; (e) residual based on band-pass filter; (f) residual based on the proposed method. 


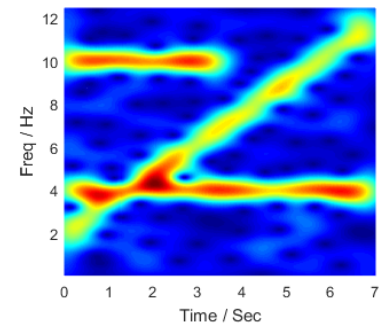

(a)

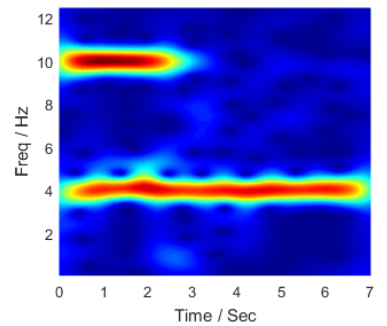

(b)

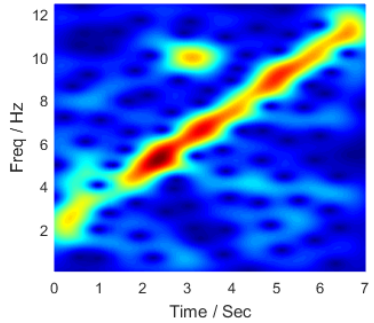

(c)

Figure 4: NLFM for example 2: (a) Time-frequency representation of origimal signal $S(t)$; (b) estimated $s_{2}$ and $s_{3} ;$ (c) remainder after removing estimated $s_{2}$ and $s_{3}$. 


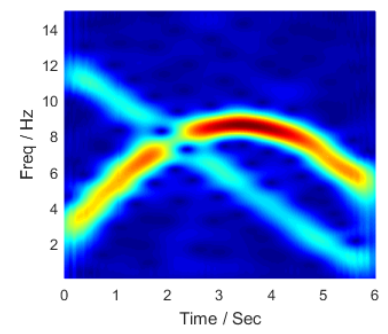

(a)

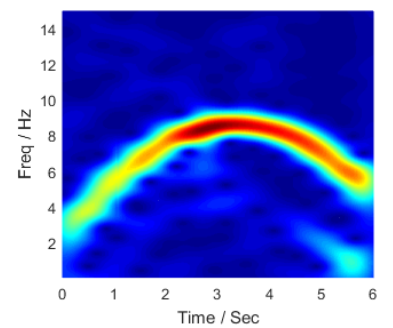

(b)

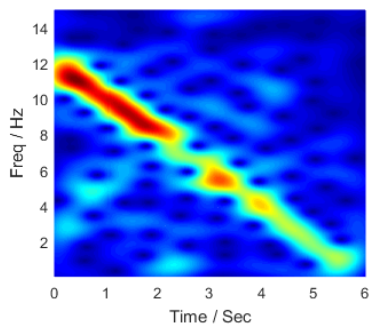

(c)

Figure 5: NLFM for example 3: (a) time-frequency representation of $S(t)$; (b) reconstructed $s_{1}$; (b) residual after removing the reconstructed $s_{1}$.

with

$$
\begin{aligned}
s_{1}(t) & =\cos \left[2 \pi\left(2 t+2 t^{2}-0.2 t^{3}\right)\right] \\
s_{2} & =0.05 \cos \left[2 \pi\left(12 t-t^{2}\right)\right]
\end{aligned}
$$

where $s_{1}(t)$ is a polynomial phase signal with the IF of $2+4 t-0.6 t^{2}$, and $s_{2}(t)$ is a chirping component with a chirp rate of $-2 \mathrm{~Hz} / \mathrm{s}$. Fig.5(a) shows these two components are overlapped in the time-frequency domain at around $t=2 s$. As the amplitude of $s_{1}(t)$ is the higher of the two components, we first isolate this component. Fig. 5(b) and (c) show a time-frequency representation of the reconstructed $s_{1}(t)$ and the residual, respectively. It is evident that the reconstructed $s_{1}$ correctly characterises the amplitude at the cross-point. It is worth noting that Fig. 5(b) shows that the lower-right of $s_{2}(t)$ is also included in the reconstructed signal. This is because the chirping rate of $s_{2}$ is similar to that of $s_{1}$ locally. In addition, we note that the energy reduction of $s_{2}$ at the cross-point in Fig. 5(c) results from the phase coupling. 


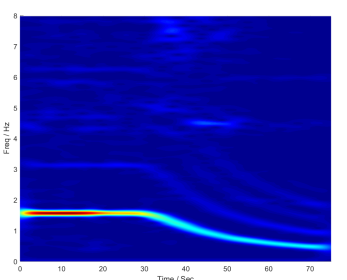

(a)

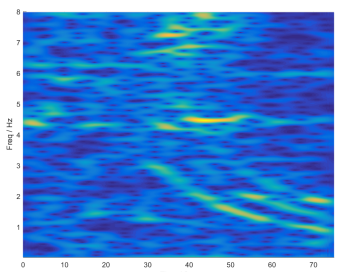

(c)

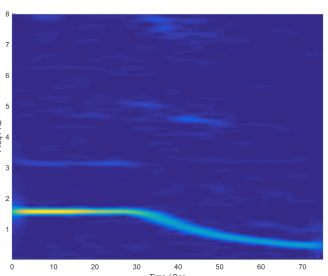

(b)

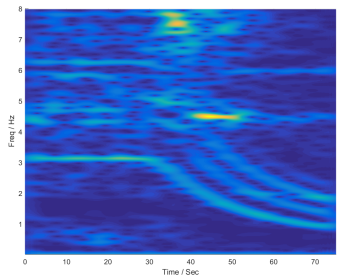

(d)

Figure 6: Example 4: (a) time-frequency plot of hydraulic turbine vibration signal; (b) remodulated component based on NLFM; (c) remainder after subtracting remodulated components based on NLFM estimation; (d) remainder after subtracting remodulated components based on BPF estimation.

\subsection{Example 4}

Finally, a vibration time-series acquired form a hydroturbine in a hydroelectric power station was studied. Fig. 6(a) shows the vibration signal $s(t)$, where the hydroturbine operates on a stationary phase from 0 to $30 \mathrm{~s}$, and shuts down afterwards. Fig. 6(b) shows the reconstructed components obtained by the proposed method. For the purposed of comparison, the residual after removing the reconstructed components based on the proposed method and the band-pass filtering are shown in Fig. 6(c) and (d), respectively. In Fig. 6(d), a belt close to fundamental component is removed, while the entire fundamental component and second-order harmonic at stationary stage were removed as shown in Fig. 6(c). Comparatively, the latter shows much richer components than original signal because they are strongly suppressed by the fundamental and second harmonic components. In addition, it is observable in Fig. 6(c) that the components at higher frequency remain in the residual. This can be explained that these components are noise instead of higher-order harmonics as expected. 


\section{Conclusion}

In this study, we performed component isolation using a framework consisting of PTFT-based demodulation and the NGLF model. An advantage of the NGLF model is that it requires no information about the number of the components to be isolated, which enables the proposed method to isolate the components that potentially have the same higher-order FM characteristics. The proposed method is suitable for multi-component signals with intersecting stationary or non-stationary components. Simulations verify that the proposed method manages to recover the amplitude of isolated components at cross-points and that it can reveal phase-coupling effects. Analysis on real data shows that the proposed method is effective for isolating stationary components and for recovering non-stationary components. Computational efficiency can be improved by applying a variational inference procedure for the IBP or by using a sliding window without overlap. Future research

will be directed towards the exploration of the NGLF model on time-series directly instead of assembling the segments as independent examples via preprocessing.

\section{Acknowledgement}

Y. Y gratefully acknowledges the support of a K.C.Wong Fellowship, the National Natural Science Foundation of China (NSFC) for Distinguished Young Scholars under Grant 11125209 and the NSFC under Grant 11472170, 51421092, 11402144. DAC was supported by the Royal Academy of Engineering, and the EPSRC via a "Grand Challenge" award.

\section{Reference}

[1] J. Urbanek, T. Barszcz, and J. Antoni, " Time-frequency approach to extraction of selected second-order cyclostationary vibration components for varying operational conditions", Measurement, vol. 46, no. 4, pp. 1454-1463, 2013.

[2] J. Han, Q. Wang, and K. Y Q. "The non-stationary signal of timefrequency analysis based on fractional Fourier transform and WignerHough transform", Mechatronics and Automatic Control Systems. Springer International Publishing, pp. 1047-1054, 2014. 
[3] S. H. Jensen, P. C. Hansen, S. D. Hansen, and J. A. Sorensen, "Reduction of broad-band noise in speech by truncated QSVD", IEEE Transactions on Speech and Audio Processing, vol. 3, no. 6, pp. 439-448, 1995.

[4] Z. Feng, S. Qin, and M. Liang, "Timefrequency analysis based on VoldKalman filter and higher order energy separation for fault diagnosis of wind turbine planetary gearbox under nonstationary conditions", Renewable Energy, vol. 85, pp. 45-56, 2016.

[5] Y. Yang, X. Dong, Z. Peng, W. Zhang, and G. Meng, "Component extraction for non-stationary multi-component signal using parameterized de-chirping and band-pass filter", IEEE Signal Processing Letters, vol. 22, no. 9, pp.1373-1377, 2015.

[6] D. P. Mandic, N ur Rehman, Z. Wu, N. E. Huang, "Empirical mode decomposition-based time-frequency analysis of multivariate signals: the power of adaptive data analysis", IEEE Signal Processing Magazine, vol. 30, no. 6, 74-86, 2013.

[7] S. Fitzgibbon, D. DeLosAngeles, T. Lewis, D. Powers, T. Grummett, E. Whitham, L. Ward, J. Willoughby, and K. Pope, "Automatic determination of EMG-contaminated components and validation of independent component analysis using EEG during pharmacologic paralysis", Clinical Neurophysiology, vol. 127, no. 3, pp. 1781-1793, 2016.

[8] H. Santosa, M.J. Hong, S.P. Kim and K.S. Hong, "Noise reduction in functional near-infrared spectroscopy signals by independent component analysis". Review of Scientific Instruments, vol. 84, no. 7, 073106, 2013.

[9] F. Nesta and O. Maurizio, "Convolutive underdetermined source separation through weighted interleaved ICA and spatio-temporal source correlation", In International Conference on Latent Variable Analysis and Signal Separation, pp. 222-230. Springer Berlin Heidelberg, 2012.

[10] P. Parathai, W. Woo, S. Dlay, and B. Gao, "Single-channel blind separation using L1-sparse complex non-negative matrix factorization for acoustic signals", The Journal of the Acoustical Society of America, vol. 137, no. 1, pp. EL124-EL129, 2015.

[11] J. Portilla, V. Strela, M. J. Wainwright, and E. P. Simoncelli, "Image denoising using scale mixtures of Gaussians in the wavelet domain", 
IEEE Transactions on Image processing, vol. 12, no. 11, pp. 1338-1351, 2003.

[12] Z. Ghahramani and T. L. Griffiths, "Infinite latent feature models and the Indian buffet process", Advances in neural information processing systems, pp. 475-482, 2015.

[13] J. Bernardo, M. Bayarri, J. Berger, A. Dawid, D. Heckerman, A. Smith, and M. West, "Bayesian nonparametric latent feature models", Bayesian Statistics, vol. 8, pp. 1-25, 2007.

[14] G.Nowak, T.Hastie, J.R. Pollack and R. Tibshirani, "A fused lasso latent feature model for analyzing multi-sample aCGH data", Biostatistics, p.kxr012, 2011.

[15] K. Miller, M.I. Jorda, and, T.L. Griffiths, "Nonparametric latent feature models for link prediction", In Advances in neural information processing systems (NIPS), Vancouver, B.C., Canada, pp. 1276-1284, 2009.

[16] A. Zhang and J. Paisley, "Markov Latent Feature Models", In Proceedings of the 33rd International Conference on Machine Learning, New York, pp. 1129-1137, 2016.

[17] S.J. Gershman, P.I. Frazier and D.M. Blei, "Distance dependent infinite latent feature models", IEEE transactions on pattern analysis and machine intelligence, vol. 37, no.2, pp.334-345, 2015.

[18] Z.Feng, X.Chen, M. Liang and F. Ma, "Time-frequency demodulation analysis based on iterative generalized demodulation for fault diagnosis of planetary gearbox under nonstationary conditions", Mechanical Systems and Signal Processing, vol.62, pp.54-74, 2015.

[19] J. Shi, M. Liang, D.S. Necsulescu and Y. Guan, "Generalized stepwise demodulation transform and synchrosqueezing for time frequency analysis and bearing fault diagnosis", Journal of Sound and Vibration, vol. 368, pp.202-222, 2016.

[20] T. L. Griffiths, and Z. Ghahramani, "The indian buffet process: An introduction and review", The Journal of Machine Learning Research, vol. 12, pp. 1185-1224, 2011. 
[21] Y. Yang, Z.K. Peng, X.J. Dong, W.M. Zhang and G. Meng, "General parameterized time-frequency transform", IEEE Transactions on Signal Processing, vol. 62, no. 11, pp.2751-2764, 2014.

[22] E. Sejdi, I. Djurovi and J. Jiang. "Time-frequency feature representation using energy concentration: An overview of recent advances", Digital Signal Processing, vol. 19, no. 1, pp. 153-183, 2009

[23] Shiqian C, Y. Yang, K.X. Wei, et al. "Time-varying frequencymodulated component extraction based on parameterized demodulation and singular value decomposition", IEEE Transactions on Instrumentation and Measurement, vol. 65, no. 2 pp. 276-285,2016.

[24] S.Q. Cheng, Z.K. Peng, Y. Yang, et al. "Intrinsic chirp component decomposition by using Fourier series representation", Signal Processing, 2017. (http://dx.doi.org/10.1016/j.sigpro.2017.01.027)

[25] Y.Yang, X.J.Dong, W.M.Zhang, et al. "Component extraction for nonstationary multi-component signal using parameterized de-chirping and band-pass filter", IEEE Signal Processing Letters, vol. 22, no. 9, pp, 1373-1377, 2015 